\title{
DEVELOPMENT OF FASCIOLA GIGANTICA EXPERIMENTALLY IN EGYPTIAN RABBITS
}

\section{By}

MOHAMED M. EI-BAHY ${ }^{1 *}$, MORSY RATEB GENEIDY ${ }^{2}$, EMAN M. H. MÉABED ${ }^{3}$, AND EMAN K. A. BAZH ${ }^{4}$

Department of Parasitology, Faculty of Veterinary Medicine, Cairo university ${ }^{1}$,

Department of Parasitology, Faculty of Medicine, Al-Azhar University², Cairo,

Departments of Parasitology, Faculty of Medicine, Fayoum University ${ }^{3}$ and

Department of Pathology \& Parasitology, Faculty of Veterinary Medicine, Damanhour

University ${ }^{4}$, Egypt ( ${ }^{*}$ Correspondence:drmelbahy@yahoo.com)

\section{Abstract}

After infection of three groups of rabbits by 50,25 and $10 \mathrm{~F}$. gigantica encysted metacercariae (EMC) for each animal, a rabbit appears as an unsuitable host for the development of this species of flukes. Rabbits need special precautions to help the worms to reach maturity before death of the infected animals. The juveniles wander in the liver tissue away from the main bile ducts, and may accidentally emerge back to the peritoneal cavity and can remain there or return back by chance to the liver. Survival of the infected rabbits, the degree of pathogenisty produced and the number of extracted worms are affected by the dose of the EMC and the general health conditions of the rabbit. Infective dose (ID) of 50 EMC led to mortalities from $28^{\text {th }}$ to $58^{\text {th }}$ day post infection (dpi.). This was accompanied by detection of flukes in the peritoneal cavity, and the anterior cones of several worms were found penetrating the liver toward the abdominal cavity. At $58^{\text {th }}$ dpi, dead animals showed marked liver cirrhosis and adhesions with the surrounding organs. Decreasing the ID to 25 EMC increased the rabbits survived till 74th dpi. The liver looked enlarged and juveniles successfully reached the main bile ducts.

Successful fluke development, with low adverse changes in the liver appearance was recorded in the group infected by $10 \mathrm{EMC}$, and the animals survived till $98^{\text {th }} \mathrm{dpi}$. The problem, at this low ID, was the decreasing in the infection rate in the exposed rabbits to $37.5 \%$. In all groups, using ELISA technique, IgM \& IgG anti-Fasciola antibodies (AFAb) increased gradually in sera. Higher titters were recorded in the group infected by 25 EMC. Prolonged migration and retardation of $F$. gigantica growth in the infected rabbits associated with either a high or a low titters. this means that the produced AFAb lack the capacity to mediate responses that can influence parasite survival. So failure of development of $F$. glgantlca growth in rabbit appears to be related mainly to its innate resistance against this Fasciola sp.

Key words: $F$. gigantica, Rabbit, Pathology, Immunology.

\section{Introduction}

Using of small non-expensive animal model was considered as an important cost benefit point in screening of new drugs or even carrying trial of different dose of vaccine. Rabbits are commonly used laboratory animals. Several authors described the successful experimental infection of rabbits with $F$. hepatica and development of worms up to egg laying (Sherif, et al, 2001, Adedokun and Fagbemi, 2001; Abdolahi and Shahriari 2016). Mango et al. (1972) reported that East African $F$. gigantica couldn't reach sexual maturity in mice, rats, hamsters, Guinea pigs and rabbits infected by dose of $5 \&$ 25 EMC/rabbit, with rabbits and rats the least susceptible ones, they added that $F . g i$ gantica in rabbits were dead and calcified by immune protection. Gerber et al. (1974) inoculated 30 rabbits with $F$. gigantica 20 metacercariae, found that on the $21^{\text {st }}$ day post infection about $50 \%$ were infected by young flukes. After 10-12 weeks the flukes developed in length but did not reach fertility but, infected rabbits suffered from disturbances of general health status, sever necrotic liver damage and died. They concluded that rabbits were not suitable model for $F$. gigantica but a useful animal to check the infectivity of metacercariae and to screen anthelmintics against flukes young stages. On the other hand, Smyth (1994) found that 
after infection of rats by $F$. hepatica, the rat's antibody-mediated cytotoxicity responses killed larval stages and young flukes as they migrate across the body cavity and through the liver. $\mathrm{He}$ added that sheep also, responded immunologically and produced anti-fluke antibodies, but these antibodies appeared to lack the capacity to mediate responses that can influence parasite survival. But, with $F$. gigantica, there was no data about its development till egg deposition in rabbit except three Egyptian papers. These were Ezzat and Abdel Ghani (1960), Hassanain et al. (1990) and EISayad (1997) who infected rabbits by 30 EMC of $F$. gigantica and detected mature flukes that gave up to $180 \mathrm{EPG}$ (egg/gm feces).

Around this wide deference between the local and foreign studies as regard susceptibility of rabbits to $F$. gigantica, and the real need of a small economic animal model for accurate evaluation of new fasciolicidal drug or vaccine, or even trials of different doses of vaccine, the present study aimed to evaluate rabbit as a non-expensive laboratory animal model for our native $F$. gigantica.

The study aimed to clarify the condition under which rabbits can survive till $F$. gigantica reached sexual maturity. This was done by infecting rabbits with different doses of EMC and evaluated the effect of the dose on the fluke survival in the infected rabbit, changes in liver, fluke age and size. Also to clarify the role of antibodies produced post infection on retardation of juvenile growth and migration outside the liver or this related to rabbit innate resistance.

\section{Material and Methods}

This study was designed and approved by Faculty of Veterinary Medicine, Cairo University Ethics Committee and was performed in accordance with the ethical standards laid down in the 1964 Declaration of Helsinki, and that of Faculty of Medicine, AlAzhar University. The study was carried out from April to September 2017.

Preparation of EMC: $F$. gigantica EMC were collected from laboratory breed Lymnaea natalensis 25-28 dpi of each snail by 4-5 miracidia under laboratory conditions. These miracidia hatched after incubation of $F$. gigantica eggs isolated from gall bladder of freshly slaughtered $F$. gigantica infected buffaloes (EI-Bahy et al, 2014). EMC were collected and stored on cellophane sheet at $4{ }^{\circ} \mathrm{C}$ till used after 5-8 days.

Infection: Thirty-two white New Zealand rabbits (1.5-2kg each) divided into 4 groups of eight rabbits each. Each rabbit in GI was infected by $50 F$. gigantica 8 days old EMC, rabbits in GII were infected each by 25 EMC, and in GIII were infected by 10 EMC each. GIV were kept as non-infected control. Before infection, rabbits were fastened for $24 \mathrm{~h}$, ID of EMC were placed on a cellophane sheet, enclosed inside lettuce and were put over the roots of rabbits' tongues. The mouth was kept closed by hand and observed for another half an hour to ensue completed swallowing without regurgitation. Then rabbits were maintained on dry food and water until the end of the experiment.

Slaughtering time and Post-mortem examination (PM): The infected rabbits were kept to live as long as possible. PM examination was applied on any of one died naturally at any post infection time. At 120dpi (Adedokum and Fagbemi, 2001) all rabbits were slaughtered, abdominal cavities were dissected out, degree of adhesions and colour of peritoneal fluid were recorded. Viscera and liver surfaces were macroscopically inspected by a hand lens (x10). Liver was extracted out to a Petri-dish with warm distilled water $\left(35-37^{\circ} \mathrm{C}\right)$. Abdominal cavity and other visceral surface were washed three times with warm water, which was collected, sieved and examined for any worms or part of them. Rabbits' liver surfaces were inspected for necrotic migratory furrows, if present; they were dissected to extract the juvenile flukes (EI-Bahy et al, 2014). Liver was then sliced into small pieces in warm water and examined under a binuclear microscope. All worms or anterior pieces of wor- 
ms recovered from liver or peritoneum were collected, counted, compressed, fixed, stained and mounted in Canada balsam (Pritchard and Kruse, 1982). Measurements were taken by micrometre eye-piece, minimum and maximum values were recorded separately.

Serological study: ELISA was applied to evaluate the antibody response to $F$. giganti$c a$ experimental infection in rabbits in relation to dose and time PI.

Serum samples: Blood samples (1-3ml) were collected from ear vein of each rabbit (infected and control) at 1, 2, 4, 6, 8, \& 10 weeks PI., left to clot and sera were stored.

Antigen used: $F$. gigantica excretory secretary antigens (ES) was prepared (Abdolahi and Shahriari, 2016). Fresh worms extracted from an infected buffalo gall bladder and washed 3 times in PBS, were incubated in PBS (pH 7.2, 1worm $/ 5 \mathrm{ml}$ ) for $3 \mathrm{hrs}$ at $37^{\circ} \mathrm{C}$. The supernatant was isolated by cool centrifugation at $10000 \mathrm{rpm}$ for one hr. Antigenic protein content was determined after Lowry et al. (1951), allocated into $1 \mathrm{ml}$ vials and stored at $-20^{\circ} \mathrm{C}$ until needed.

Reference control sera (rabbit hyperimmune sera, HIRS): Three (2 months old) white New Zealand rabbits, parasite-free, maintained under specific pathogen-free condition with suitable humidity and temperature, at Department of Parasitology, Veterinary Medicine, Cairo University. HIRS were prepared (Tang et al, 2015) with modification. Pre-immune sera were collected before immunizations (as negative control). Rabbits were subcutaneously immunized with $1.2 \mathrm{mg}$ protein of ES Ag, emulsified 1:1 (v/v) with mineral oil as adjuvant, followed by three boost immunizations at 2-week intervals with $0.4 \mathrm{mg}$ protein with same adjuvant. Two weeks after the last immunization, rabbits were bled from the ear vein for serum collection. The sera were stored at $-20^{\circ} \mathrm{C}$ until used as positive control.

ELISA evaluated the antibodies against $F$. gigantica developed in rabbit in relation to dose and time post infection (PI.) after Liu et al. (2015) with little modification. Each plate was coated over night with antigens at optimal dilution $4 \mathrm{ug} / \mathrm{ml}$ in coating buffer (optimal dilutions of various reagents were determined by checkerboard titration.). After washing, sera and reference control hyperimmune sera at 1:100 dilutions were added (100ul/well), incubation at $37^{\circ} \mathrm{C}$ for $90 \mathrm{~min}-$ utes, and plate was washed and goat anti-rabbit IgM \& IgG horseradish peroxidase conjugate (Sigma) were used at 1:2000 dilution. Ortho-phenylenediamidine-OPD was added as $340 \mathrm{ug} / \mathrm{ml}$ substrate buffer. Absorbance was read at $450 \mathrm{~nm}$ using a Titerteck multiskan ELISA reader. Positive ELISA optical density $=$ mean value of negative control +2 folds of standard deviation. Sera of 5 rabbits on same day PI and 5 controls were tested separately, two wells for each. Mean of each $\&$ five rabbits were recorded as one value. Positive ELISA was compared with control on same time PI (Liu et al, 2015).

\section{Results}

Infection of 8 rabbits each by $50 F$. gigantica EMC GI showed a marked dangerous effect on liver and general health conditions causing death within 58 d.p.i. P.M. examination of two rabbits died at $28^{\text {th }} \& 30^{\text {th }}$ d.p.i. showed liver surface creamy colour with pink rounded spots $1-2 \mathrm{~mm}$ in diameter and short streaks up to $7 \times 1-2 \mathrm{~mm}$ specially on right lobe periphery close to duodenum. Anterior cones of two worms penetrated liver surface towards abdominal cavity. Extra-hepatic, bile ducts were normal without gross peritonitis on surface. Two small worms of $3.5 \times 1.0 \mathrm{~mm} \& 5 \times 1.5 \mathrm{~mm}$ were detected in peritoneal wash of rabbit died at $30^{\text {th }}$ d.p.i.

Liver maceration after that gave 8 \& 5 worms little longer than that of peritoneum (6.4-7.6x 1.0-1.6mm). The rest of the infected rabbits died at $56^{\text {th }}$ d.p.i. (2), $57^{\text {th }}$ d.p.i. (one) and $58^{\text {th }}$ d.p.i.. (3). During these 3 days, 19 juvenile flukes were extracted from liver (size ranged from 13.3-14.6x1.2-1.6 $\mathrm{mm}$ ) and 10 worms were in the peritoneal wash (size ranged from 9-11x1-1.4mm) The livers were enlarged firm in consistency, irregular surface, with a thick superficial co- 
vering layer of firmly adherent fibrin, whitish in colour connecting liver to surrounding organs. This layer caused firmly adhesion between the liver lobes, unusual pale colour, grossly enlarged $(79 \mathrm{gm})$ and firm in consistency. Slaughtering of two controls on same days showed liver normal smooth glistening with weight of $54 \mathrm{gm}$.

Peritoneal cavities of infected rabbits died at $56^{\text {th }}$ to $58^{\text {th }}$ d.p.i. showed whitish fibrous substances with peritoneal fluid in-between. Rabbits were severely emaciated. Internal organs and muscles were pale in colour with poorness symptoms. Gall bladder was not grossly enlarged but bile was dark brown in colour. Some worms were more superficially at the periphery of the lobs clearing either migration to or from peritoneal cavity with low sensitivity towards bile ducts.

With decreasing EMC dose to 25/rabbit was the time of first mortality delayed to $58^{\text {th }}$ d.p.i. (3), then at $73^{\text {th }}$ d.ph.i. (1) but 4 died at $74^{\text {th }}$ d.p.i. Those died at $85^{\text {th }}$ d.p.i. didn't have severe inflammation or adhesion recorded at same time in G1. Liver was little enlarged, with nearly smooth surface. Three worms were found in peritoneal wash and 12 were extracted from liver tissue near surface lesion. One rabbit died at $73^{\text {rd }}$ d.p.i. showed marked lesion of worm penetration on liver surface but none was extracted after maceration. At $74^{\text {th }}$ d.p.i. four rabbits died from infection were emaciated and weak on day $50^{\text {th }}$ p.i.

The liver enlarged with smooth surface (68 gm), capsule was transparent with neither rough thick fibrin on surface nor adhesion with surrounding organ. Fourteen worms were extracted livers, with length varied from 14.5-16.5x2-3mm. Those extracted from the main bile duct near the hilus region of $2 /$ rabbits were in peritoneal wash, but none in peritoneal cavity. The worms were smaller (12.5-14.5x2-3mm). Two controls slaughtered at $74^{\text {th }}$ day had normal smooth brownish liver with mean weight of $56 \mathrm{gm}$.

Details were given in tables $(1,2, \& 3)$ and figures $(1 \& 2)$.

Table 1: Infection and frequency of mortality in rabbits infected by different doses of EMC:

\begin{tabular}{|c|c|c|c|c|c|}
\hline Infected Rabbits & Time of death or slaughtered & Dead rabbits & $\%$ & Infected rabbits & $\%$ infected \\
\hline \multirow{5}{*}{$\begin{array}{l}\text { G1 } \\
50 \text { EMC }\end{array}$} & $28^{\text {th }} \mathrm{dpi}$ & 1 & 12.5 & \multirow{5}{*}{$8 / 8$} & \multirow{5}{*}{$100 \%$} \\
\hline & $30^{\text {th }}$ dpi & 1 & 12.5 & & \\
\hline & $56^{\text {th }}$ dpi & 2 & 25.0 & & \\
\hline & $57^{\text {th }}$ dpi & 1 & 12.5 & & \\
\hline & $58^{\text {th }} \mathrm{dpi}$ & 3 & 37.5 & & \\
\hline \multirow{3}{*}{$\begin{array}{l}\text { G2 } \\
25 \text { EMC }\end{array}$} & $58^{\text {th }} \mathrm{dpi}$ & 3 & 37.5 & \multirow{3}{*}{$8 / 8$} & \multirow{3}{*}{$100 \%$} \\
\hline & $73^{\text {rd }}$ dpi & 1 & 12.5 & & \\
\hline & $74^{\text {th }} \mathrm{dpi}$ & 4 & 50.0 & & \\
\hline \multirow{3}{*}{$\begin{array}{l}\text { G3 } \\
10 \text { EMC }\end{array}$} & $96^{\text {th }} \mathrm{dpi}$ & 2 & 25.0 & \multirow{3}{*}{$5 / 8$} & \multirow{3}{*}{$62.5 \%$} \\
\hline & $98^{\text {th }}$ dpi & 3 & 37.5 & & \\
\hline & $120^{\mathrm{th}} \mathrm{dpi}$ & $3 *$ & 37.5 & & \\
\hline
\end{tabular}

No mortalities or worms extracted from control non infected rabbits slaughtered in corresponding days of mortality of others

Table 2: Size range of extracted flukes and rate of infection

\begin{tabular}{|c|c|c|c|c|c|c|c|c|c|}
\hline \multirow{2}{*}{$\begin{array}{l}\text { Rabbit } \\
\text { groups }\end{array}$} & \multirow{2}{*}{$\begin{array}{l}\text { Death or slau- } \\
\text { ghtering tine }\end{array}$} & \multicolumn{3}{|c|}{ From Liver } & \multicolumn{3}{|c|}{ From Peritoneum } & \multirow{2}{*}{$\begin{array}{l}\text { fluke/ } \\
\text { rabbit }\end{array}$} & \multirow{2}{*}{$\begin{array}{l}\text { fluke/ } \\
\text { EMC }\end{array}$} \\
\hline & & No. & Length & Breadth & No. & Length & Breadth & & \\
\hline \multirow{5}{*}{ G1 } & $28^{\text {th }} \mathrm{dpi}$ & 8 & \multirow{2}{*}{$6.4-7.6$} & \multirow{2}{*}{$1-1.6$} & - & \multirow{2}{*}{$3.5-5$} & \multirow[b]{2}{*}{$1-1.5$} & \multirow{5}{*}{5.5} & \multirow{5}{*}{$11.0 \%$} \\
\hline & $30^{\text {th }}$ dpi & 5 & & & 2 & & & & \\
\hline & $56^{\text {th }} \mathrm{dpi}$ & 9 & \multirow{3}{*}{$13.3-14.6$} & \multirow{3}{*}{$1.2-1.6$} & 3 & \multirow{3}{*}{$9-11$} & \multirow{3}{*}{$1.1-1.4$} & & \\
\hline & $57^{\text {th }}$ dpi & 3 & & & 1 & & & & \\
\hline & $58^{\text {th }} \mathrm{dpi}$ & 7 & & & 6 & & & & \\
\hline \multirow{3}{*}{ GII } & $58^{\text {th }} \mathrm{dpi}$ & 12 & $12.5-14$ & $1.8-2$ & 3 & 12 & $2-3$ & \multirow{3}{*}{4.125} & \multirow{3}{*}{$16.5 \%$} \\
\hline & $73^{\text {rd }} \mathrm{dpi}$ & 0 & & & 0 & & & & \\
\hline & $74^{\text {th }} \mathrm{dpi}$ & 14 & $14.5-16.5$ & $2-3$ & 4 & $12.5-14.5$ & $2-3$ & & \\
\hline \multirow{3}{*}{ GIII } & $96^{\text {th }}$ dpi & 7 & $17.5-23.5$ & $3-4.2$ & - & & & \multirow{3}{*}{3} & \multirow{3}{*}{$18.75 \%$} \\
\hline & $98^{\text {th }} \mathrm{dpi}$ & 8 & $18-25$ & $3-4.2$ & - & & & & \\
\hline & $120^{\text {th }} \mathrm{dpi}$ & 0 & & & & & & & \\
\hline
\end{tabular}

In GIII as each one was exposed to 10 EMC/rabbit, they were still surviving for 
long time (96-98 d.p.i.) without marked disturbances. Emaciation and weakness appeared on infected rabbits from the day $65^{\text {th }}$ p.i. Three rabbits from this group $(37.5 \%)$ did not die, did not show marked change in their general health conditions and still alive.On slaughtering them at $120^{\text {th }}$ d.p.i. they appeared to be overcome this dose level after penetration of the juvenile flukes, where very minute lesions were detected on their liver surface without extraction of any worm after maceration of their liver.

The liver of rabbits died at $96 \& 98^{\text {th }}$ d.p.i. appeared as that in fig (1-A), have normal colour, glistening, and consistency. Its mean weight was 57 gram (mean weight of 2 livers from normal non-infested rabbits of similar age was 58 gram). The only lesions detected on their liver surface was a round elevated necrotic points yellowish to brown in colour representing the site of juvenile worms penetration. There was no adhesion or fibrosis between the liver and the surrounding organs that have a normal colour. Two to three worms were extracted from the main bile ducts of each liver. The extracted flukes were larger than before measured 18$26 \mathrm{~mm}$ length and 3-4.2 mm breadth, in a good nourished condition. The mounted specimens showed complete development of most internal organs but there was no egg present in worms' uterus to day $98^{\text {th }}$ d.p.i. Only 4 eggs of Fasciola species were small elongated, without embryonic cells and measured $110-125 \mathrm{x} 45 \mathrm{u}$, were detected in examining warm water of a rabbit died at $98^{\text {th }}$ p.i. after liver maceration. No worm was detect- ed in peritoneal wash at PI. The infection rate was $100 \%$ in rabbits exposed to $F$. gigantica in dose of 25-50 EMC/rabbit, but was $62.5 \%$ with $10 \mathrm{EMC} / \mathrm{rabbit}$. There was a direct relation between of EMC dose increases and early mortalities.

Level of antibodies developed in infected rabbits: The level of AFAb (IgM \& IgG) using ELISA was estimated in relation to infection by different doses of EMC as described. The data revealed gradually increase in IgM level directly after infection during the first week post infection (wpi), little decreased during the second wpi and then disappeared. Antibody titer reflexed by ELISA/OD was high in rabbits infected by high dose (0.735) than those infected by low dose (0.622) as compared to control (0.023).

$\mathrm{IgG}$ against juveniles increased from $2^{\text {nd }}$ w.p.i., at $3^{\text {rd }}$ wpi, value was higher in GI (0.681) than GII $(0.677)$ or GIII $(0.598)$

ELISA OD was increased in GI to 0.655 and 0.671 at the $4^{\text {th }}$ and $6^{\text {th }}$ wpi. respectively and did not increase but it decreased to 0.555 after this till end of the observation period which was associated with death of these rabbits at $58^{\text {th }}$ d.p.i. In the contrary, mean ELISA/OD was increased in the other two groups for $0.733,0.700,0.656$ and 643 in GII and it reached to $0.811,0.786,0.702$ \& 0.632 in GIII at the weeks $4,6,8 \& 10$ post infections respectively in comparison with GI and control group. The data showed no marked role for the increase in the dose of EMC in causing severe changes in the level of produced anti-Fasciola antibodies on the level of present study

Table 3: Change in mean ELISA values in relation to dose of $F$. gigantica EMC and post infection time.

\begin{tabular}{|c|c|c|c|c|c|c|c|c|}
\hline \multirow{2}{*}{$\begin{array}{l}\text { Dose of } \\
\mathrm{EMC} / \mathrm{rabbit}\end{array}$} & \multirow{2}{*}{ Ig } & \multicolumn{7}{|c|}{ Mean ELISA value for 5 rabbit samples at, } \\
\hline & & $1^{\text {st }}$ wpi & $2^{\text {nd }}$ wpi & $3^{\text {rd }}$ wpi & $4^{\text {th }}$ wpi & $6^{\text {th }}$ wpi & $8^{\text {th }}$ wpi & $10^{\text {th }}$ wpi \\
\hline \multirow{2}{*}{$\begin{array}{l}\text { G.I } \\
(50 \text { EMC) }\end{array}$} & $\operatorname{IgM}$ & 0.735 & 0.555 & 0.033 & 0.023 & 0.022 & 0.024 & 0.022 \\
\hline & $\mathrm{IgG}$ & 0.117 & 0.421 & 0.681 & 0.655 & 0.671 & 0.644 & 0.555 \\
\hline \multirow{2}{*}{$\begin{array}{l}\text { G.1I } \\
(25 \text { EMC) }\end{array}$} & $\operatorname{IgM}$ & 0.665 & 0.523 & 0.040 & 0.035 & 0.030 & 0.027 & 0.028 \\
\hline & IgG & 0.115 & 0.446 & 0.677 & 0.733 & 0.700 & 0.656 & 0.643 \\
\hline \multirow{2}{*}{$\begin{array}{l}\text { G.-III } \\
(10 \text { EMC) }\end{array}$} & $\operatorname{IgM}$ & 0.622 & 0.456 & 0.030 & 0.025 & 0.027 & 0.022 & 0.026 \\
\hline & $\operatorname{IgG}$ & 0.116 & 0.567 & 0.598 & 0.811 & 0.786 & 0.702 & 0.632 \\
\hline \multirow{2}{*}{$\begin{array}{l}\text { Control } \\
\text { (non infected) }\end{array}$} & $\operatorname{IgM}$ & 0.023 & 0.021 & 0.026 & 0.025 & 0.021 & 0.025 & 0.024 \\
\hline & IgG & 0.011 & 0.013 & 0.014 & 0.012 & 0.011 & 0.015 & 0.014 \\
\hline
\end{tabular}

wpi $=$ weeks post infection 


\section{Discussion}

Development of Fasciola in the vertebrate hosts was affected by multiple factors control the infective dose (ID) and the host innate resistance. The two factors controlled the suspected pathogenicity that occurred in this host (Mango et al, 1972). This work developed during continuous observation recorded among others work on $F$. gigantica in rabbits. This agreed with Gerber et al. (1974) Adedokun and Fagbemi (2001) and Sherif et al. (2001), but differed with Hassanain et al. (1990).

Infection of three groups of rabbits by 3 different doses of $F$. ggiantica EMC proved rabbit as a host but with special condition to for maturity before rabbit death. Development of $F$. ggiantica in rabbit affected by EMC, depended on rabbit health condition, innate resistance, management and nutrition post infection. The juveniles that penetrate liver early in their life returned back to peritoneum around $28^{\text {th }}$ dpi., which caused mortality in weak rabbit (Gerber et al, 1974).

In the present study, $50 \mathrm{EMC} / \mathrm{rabbit}$ led to death of all rabbits preceded by weakness and emaciation before death, on PM there was liver cirrhosis, and adhesion with surrounding organs. Extraction of juveniles migrated superficial in liver tissues clarified that their migrated without sensitivity requirement or attraction toward bile duct. This disagreed with Adedokum and Fagbemi (2001) who found that flukes in the bile ducts 40 days after infection in sheep. With dose of 25EMC rabbits survived for long time. Their liver enlarged, degree of adhesion was less, with some flukes in peritoneal cavity and liver. With dose to 10 EMC, rabbits survived without disturbances in health conditions for long time, without worms in the peritoneal cavity. This might be due to the fact that juvenile flukes found enough room to migrate into the liver. A dose was suitable for worm development in experimentally rabbits. This agreed with Adedokun and Fagbemi (2001) and Sherif et al. (2001) who used low dose (5EMC) to obtain
$F$. gigantica in rabbit. But, this disagreed with Hassanain et al. (1990) and EI-Sayad (1997). At 5-10 EMC worm reached maturity but infection failed in some animals (Sherif et al, 2001). Failure in some rabbits was related to its' innate resistant to capsulation of early juveniles with low chemotactic sensitivity to reach bile ducts. In the present study, rabbit survived for a time with good health condition and low pathogenicity. This clarified presence of natural fascioliasis infection in rabbits (Apt et al, 1993). Migration of juvenile flukes to liver led to severe fibrosis and adhesion between liver and surrounding organs from repeated bleeding due to EMC high dose. The repeated penetration to liver due to high dose caused mortality (Srivastava and Singh, 1972).

In the present study, identification of eggs after maceration of one liver showed proved Fasciola development under experimental conditions. Also, absence worm or piece of worm with eggs in the uterus might be due to the fact that eggs originated from one early mature worm, destructed during liver maceration with presence of worms' anterior.

In the present study, IgM gradually increased in the $1^{\text {st }}$ wpi, slight decreased in $2^{\text {nd }}$ and then disappeared. $\operatorname{IgG}$ increased in $2^{\text {nd }}$ to $4^{\text {th }}$ wpi and then slightly decreased to experimental end. Changes in antibody level were not related to EMC dose used. Mild variations in level might be related to host ability to deal with infection. This agreed with Itagald et al. (1989) in cattle using $F$. hepatica, IgG was low in $1^{\text {st }}$ wpi. and then increased from the $4^{\text {th }}$ to $6^{\text {th }}$ wpi.

Decrease of the antibodies' level in GI after the $6^{\text {th }}$ wpi was related to weakness and ultra-ions in rabbits' health conditions. Doses of 10 or 25 EMC stimulated rabbit immune system to produce more antibodies without bad effect on health conditions. Absence of relation between infective dose and level of AFAb showed that rate of fluke development depended on immune system to tolerate the stress on liver infection. This 
agreed with Rickard and Howell (1982) who reported the same in sheep. But, Hughes et al. (1981) found that antibody-mediated cytotoxic action killed migrating larval stages and young flukes.

No doubt, the worms size was related to age of fluke and nutrient available to larval stages, flukes in liver were good nourished than those in peritoneum, the ectopic site. Also, fluke extracted from liver fibroses was small in size than those extracted from nonfibroses parenchymatic tissue.

\section{Conclusion}

The outcome data, recommended infecting large number of rabbits by 10EMC. This dose protected them from early death and overcome the percentage of failure of infection. Fluke supposed to reach maturity in rabbits $14-16$ weeks post infection.

\section{References}

Abdolahi, S, Shahriari, B, 2016: Detection of Fasciola hepatica and Fasciola gigantica common and uncommon antigens, using rabbit hyper immune serum raised against their excretorysecretory and somatic antigens. https://www. researchgate. net/publication/290507400_

Adedokun, OA, Fagbemi, BO, 2001: The assessment of Fasciola gigantica infection in the rabbit (Oryctolagus cuniculus) as a laboratory model parasite development clinical symptoms and liver pathology. Afr. J. Biomed. Res. 14: 161-3

Apt, W, Aguilera, X, Vega, F, Alcaíno, H, Zulantay, I, et al, 1993: Prevalence of fascioliasis in humans, horses, pigs, \& wild rabbits in $3 \mathrm{Chi}-$ lean provinces. Bol. Ofic. Sanit Panam. 115, 5: 405-14

El-Bahy, MM, Mahgoub, AM, Taher, EE, 2014: Contributions on human fascioliasis and its snail intermediate host in Nile Delta, Egypt

Int. J. Basic Applied Sci. 3, 3:172-9

EI-Sayed, MH, 1997: Comparative studies on the effect of bithionol, praziqantel and triclabendazole in rabbit's fascioliasis. J. Egypt. Soc. Parasitol. 27, 1:131-42.

Ezzat, MA, Abdel-Ghani, AF, 1960: The daily output of $F$. gigantica eggs as estimated from a naturally infected rabbit. Proceed. $1^{\text {st }}$ Ann. Vet. Cong. Egypt.

Gerber, H, Horchner, F, Oguz. A, 1974: F. gigantica infection in small laboratory animals. Berl. Munch. Tierarzt.Wschr. 87, 11: 207-10.
Hassanain, MA, Shalaby, S, Derbala, A, 1990: Infectivity and antibody response of irradiated $F$. gigantica encysted metacercaria in rabbits. J. Egypt. Vet. Med. Ass. 50, 2:267-4.

Hughes, D, Harness, E, Doy, T, 1981: The different stages of Fasciola hepatica capable of inducing immunity and the susceptibility of various stages to immunological attack in the sensitized rat. Res. Vet. Sci. 30:93-8.

Itagaki, T, Ohta, N, Hosaka, Y, Chinone, S, Itagaki, H, 1989: Diagnosis of Fasciola species infection in cattle by Enzyme-Linked ImmunoSorbent Assay. Japn. J. Vet. Sci 51, 4:23-8.

Liu, P, Cui, J, Liu, RD, Wang, M, Jiang, P, et al, 2015: Protective immunity against Trichinella spiralis infection induced by $\mathrm{TsNd}$ vaccine in mice. Parasit. Vectors, 8:185-92.

Lowry, OH, Rosebrough, NJ, Farr, AL, Rondall. RJ, 1961: Protein measurement with the Folln phenol reagent. J. Bio. Chem. 193:265-75. Mango, AM, Mango, CK, Esamal, D, 1972: A preliminary note on the susceptibility, prepatency and recovery of $F$. gigantica in small laboratory animals. J. Helminthol. 46, 4:381-6.

Oldham, G, 1983: Antibodies to $F$. hepatica antigens during experimental infections in cattle measured by ELISA. Vet. Parasitol. 13:151-8.

Pritchard, MN, Kruse, CO, 1982: The Collection and Preservation of Animal Parasites: Library of Congress Cataloguing in Publication Data.

Rickard, M, Howell, M, 1982: Comparative aspects of immunity in fascioliasis and cysticercosis in domesticated animals. In: Biology \& Co-ntrol of Endoparasites. Academic Press, Austraila. Sherif, N, Shalaby, I, Abdel Moneim, M, Soliman, M, 2001: Experimental infection of rabbits with Fasciola gigantica and treatment with triclabendazole (TCBZ) or BT6: parasitological, haematological and immunological findings. J. Egypt. Publ. Hlth. Assoc. 76, 5/6:505-29.

Smyth, JD, 1994: Introduction to animal Parasitology, $3^{\text {rd }}$ edition. Press Syndicate of the University of Cambridge, Pitt Building, Trumpington Street, Cambridge CB2 1RP, 40

Srivastava, PS, Singh, KS, 1972: Some observations on the pathology of experimental Fasciola gigantica infection in rabbit. Indian J. Anim. Sci. 42, 1:72-6.

Tang, B, Liu, M, Wang, L, 2015: Characterisation of a high-frequency gene encoding a strongly antigenic cystatin-like protein from Trichinella spiralis at its early invasion stage. Parasit. Vectors 8:78-92. 


\section{Explanation of figures}

Fig. 1: Changes in ELISA-IgM and IgG in infected rabbits according to EMC dose.

Fig. 2: Pathological changes in liver of rabbits infected by different EMC doses:

A - Liver of rabbit died at $98^{\text {th }}$ d.p.i. after infection by 10 EMC: Liver normal in color, size, with normal gall bladder necrotic foci at site of juvenile fluke penetration (arrow). B - Liver of rabbit infected by $25 \mathrm{EMC}$ died at $74^{\text {th }}$ d.p.i. Enlarged liver with less corrugated surface. Liver capsule thin transparent without thick fibrous adhesion (arrow). C - Liver of rabbit died at $58^{\text {th }}$ d.p.i. ( $\left.50 \mathrm{EMC} / \mathrm{rabbit}\right)$ : Enlarged haemorrhgic rough surface, marked lobule, and fibrins adhesion with surrounding organs. D - Premature F. gigantica extracted from liver of rabbits died at $73^{\text {rd }}$ d.p.i.(Carmine stained mounted specimen). E- One F. gigantica worm (arrow) detected more superficial protrusion its anterior cone toward the peritoneal cavity from enlarged corrugated liver at $58^{\text {th }}$ d.p.i. (from the group of 50 EMC/rabbit). F - Immature F.gigantica worms extracted from liver and peritoneum of rabbits died at $28^{\text {th }} \& 30^{\text {th }}$ d.p.i. (fresh specimen).

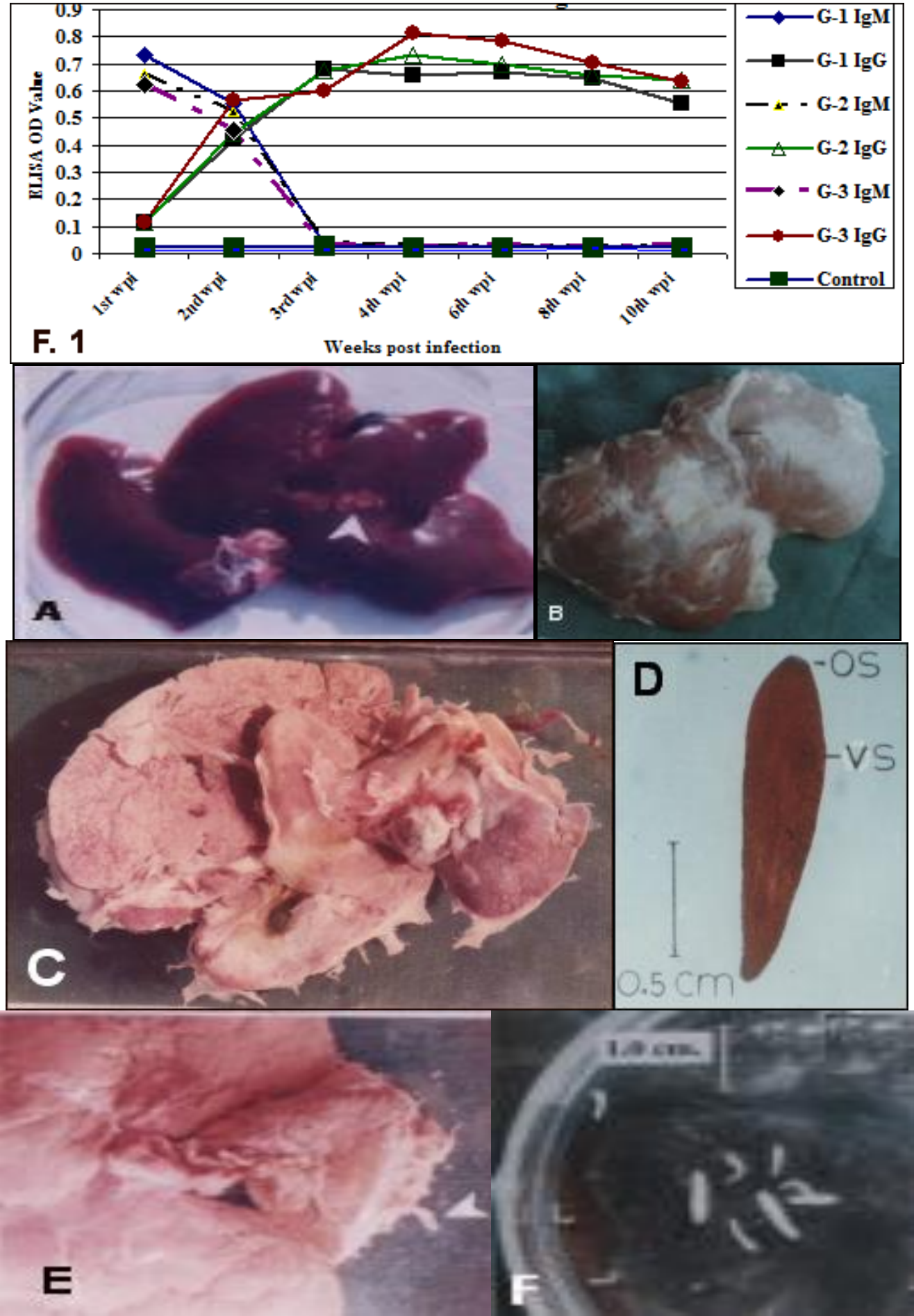

